\title{
EXTRAÇÃO SELETIVA DE CÁLCIO E MAGNÉSIO DE LICORES SULFÚRICOS CONCENTRADOS DE NÍQUEL VIA EXTRAÇÃO POR SOLVENTES*
}

Alexandre Silva Guimarães ${ }^{1}$ Marcelo Borges Mansur ${ }^{2}$

\begin{abstract}
Resumo
Investigou-se a extração seletiva de $\mathrm{Ca}$ e $\mathrm{Mg}$ do licor sulfúrico concentrado de $\mathrm{Ni}$ visando sua purificação. Observou-se que a mistura sinérgica dos extratantes D2EHPA + ácido naftênico apresenta maior afinidade na extração de $\mathrm{Ca}$ em $\mathrm{pH} \leq 5$. Comportamento oposto foi verificado pelo extratante Cyanex 272, cuja preferência de extração é pelo $\mathrm{Mg}$ para $5 \leq \mathrm{pH} \leq 6$. A cinética de extração de $\mathrm{Ca}$ e $\mathrm{Mg}$ é rápida atingindo o equilíbrio em menos de 6 minutos. A purificação do licor de $\mathrm{Ni}$ requer a utilização de 2 circuitos de extração em operações estagiadas. No primeiro circuito, Ca pode ser removido do licor empregando-se o sistema orgânico D2EHPA (10\% $\mathrm{v} / \mathrm{v})+$ ácido naftênico (20\% v/v). O pH ótimo é 3,5, no qual foram extraídos $72 \%$ de $\mathrm{Ca}\left(\beta_{\mathrm{Ca} / \mathrm{Ni}}=40\right)$ em uma contatação. No segundo circuito, o licor deve ser submetido a uma extração de $\mathrm{Mg}$ pelo Cyanex 272 (20\% v/v). O pH operacional propício é 5,5 com extração de $66 \%$ de $\mathrm{Mg}$ ( $\beta_{\mathrm{Mg} / \mathrm{Ni}}=16$, uma contatação). O licor purificado de $\mathrm{Ni}$ pode ser encaminhado à etapa de eletrorrecuperação almejando-se a obtenção de Ni metálico.
\end{abstract}

Palavras-chave: Níquel; D2EHPA; Cyanex 272; Extração por solventes.

\section{SELECTIVE EXTRACTION OF CALCIUM AND MAGNESIUM FROM CONCENTRATED NICKEL SULFURIC LIQUOR VIA SOLVENT EXTRACTION \\ Abstract}

This work investigated the selective extraction of $\mathrm{Ca}$ and $\mathrm{Mg}$ from concentrated $\mathrm{Ni}$ sulfuric liquor aiming your purification. It was observed that the synergistic mixture of the extractants D2EHPA + naphthenic acid presents higher affinity in the extraction of $\mathrm{Ca}$ at $\mathrm{pH} \leq 5$. Opposite behavior was verified by the extractant Cyanex 272, whose extraction preference is by $\mathrm{Mg}$ at $5 \leq \mathrm{pH} \leq 6$. The kinetics extraction of $\mathrm{Ca}$ and $\mathrm{Mg}$ is rapid reaching equilibrium in less of 6 minutes. Purification of $\mathrm{Ni}$ liquor requires the use of 2 extraction circuits in staged operations. In the first circuit, $\mathrm{Ca}$ can be removed from the liquor using the organic system D2EHPA $(10 \% \mathrm{v} / \mathrm{v})+$ naphthenic acid $(20 \% \mathrm{v} / \mathrm{v})$. The optimal $\mathrm{pH}$ is 3.5 , in which $72 \% \mathrm{Ca}\left(\beta_{\mathrm{ca} / \mathrm{Ni}}=40\right)$ were extracted in a single contact. In the second circuit, the liquor must be submitted to $\mathrm{Mg}$ extraction by Cyanex $272(20 \% \mathrm{v} / \mathrm{v})$. The propitious operating $\mathrm{pH}$ is 5.5 with extraction of $66 \%$ $\mathrm{Mg}\left(\beta_{\mathrm{Mg} / \mathrm{Ni}}=16\right.$, in a single contact). Purified $\mathrm{Ni}$ liquor can be directed to electrowinning to obtain Ni metal.

Keywords: Nickel; D2EHPA; Cyanex 272; Solvent extraction.

1 Engenheiro Metalurgista, Mestre, Doutorando em Engenharia Metalúrgica, Materiais e de Minas, Universidade Federal de Minas Gerais, Belo Horizonte, MG, Brasil.

2 Engenheiro Químico, Doutor, Professor Associado, Programa de Engenharia Metalúrgica e de Materiais, Universidade Federal do Rio de Janeiro, Rio de Janeiro, RJ, Brasil. 


\section{INTRODUÇÃO}

Na recuperação e purificação hidrometalúrgica de Ni via re-lixiviação do precipitado de hidróxido misto (MHP), o licor obtido contendo Ni ( 70-90 g/L) e Co ( 2-8 g/L) encontra-se impuro ( $\mathrm{Ca}, \mathrm{Cu}, \mathrm{Mg}, \mathrm{Mn}$ e $\mathrm{Zn}[1,2,3])$, sendo necessário tratamento para purificação [4], normalmente pela técnica de extração por solventes [5]. Esta consiste em colocar em contato o licor de re-lixiviação (fase aquosa) com uma fase orgânica contendo espécies reativas (extratantes) solubilizadas em um diluente orgânico apropriado [6,7]. O extratante reage com os metais de interesse formando complexos organometálicos solúveis na fase orgânica e, portanto, separados seletivamente.

Guimarães et al [8] demonstraram que Zn, Co, Cu e Mn podem ser separados do Ni, $\mathrm{Ca}$ e $\mathrm{Mg}$, em operações estagiadas, utilizando-se o extratante Cyanex 272 (ácido 2,4,4-trimetil-pentil fosfínico) na concentração de $20 \%$ v/v. Nesta condição, foram extraídos $99 \%$ de $\mathrm{Zn}$ em pH 2,5, 52\% de Co, $87 \%$ de $\mathrm{Cu}$ e $78 \%$ de $\mathrm{Mn}$ em pH 3,9 a $50^{\circ} \mathrm{C}$ e razão $\mathrm{A} / \mathrm{O}=1$ (razão entre os volumes das fases aquosa $(\mathrm{A})$ e orgânica $(O)$ ). Os metais Co, Cu e Mn são reextraídos da fase orgânica com ácido sulfúrico [3]. Após essa etapa, o refino de Co pode ser realizado combinando-se precipitação e extração por solventes; conforme Donegan [9], Co é precipitado como sulfeto, separando-o do $\mathrm{Mn}$ e, em seguida, Co e Cu são re-lixiviados e separados entre si utilizando-se o extratante D2EHPA (ácido di-2-etilhexil fosfórico) [10]. O Co purificado é, então, enviado à etapa de eletrorrecuperação obtendo-se catodos de $\mathrm{Co}$ [4]. O refino de $\mathrm{Ni}$ em relação às impurezas $\mathrm{Ca}$ e $\mathrm{Mg}$ pode ser conduzido via extração por solventes. Dessa maneira, usando-se um sistema extrativo sinérgico (mistura de dois ou mais extratantes) contendo $20 \%$ v/v de Cyanex $272+10 \%$ v/v de ácido versático (ácido neodecanóico), Guimarães et al [8] analisaram a separação $\mathrm{Ca} / \mathrm{Ni}$ e $\mathrm{Mg} / \mathrm{Ni}$ do licor, extraindo cerca de $40 \%$ de $\mathrm{Ca}, 40 \%$ de $\mathrm{Mg}$ e $6 \%$ de $\mathrm{Ni}$ em $\mathrm{pH}$ 5,1 .

Em outra oportunidade, Guimaraes et al [11] utilizaram um sistema sinérgico contendo 20\% v/v de Cyanex $272+5 \%$ v/v de Cyanex 923 (mistura de 4 óxidos trialquil fosfínicos) com o intuito de separar $\mathrm{Ca}$ e $\mathrm{Mg}$ do $\mathrm{Ni}$. Embora as extrações de $\mathrm{Mg}$ mostrassem satisfatórias (68\%) com $\beta_{\mathrm{Mg} / \mathrm{Ni}}=18 \mathrm{em} \mathrm{pH}=5,4$, esse sistema se mostrou ineficiente na extração de $\mathrm{Ca}(7 \%, \beta \mathrm{Ca} / \mathrm{Ni} \leq 0,8)$ para $4,5 \leq \mathrm{pH} \leq 6,4$. Santos et al [12] extraíram 100\% de $\mathrm{Ca}$ do licor, em $\mathrm{pH} 2$, utilizando-se sistemas aquosos bifásicos. Esta técnica, porém, foi ineficaz para promover a separação do $\mathrm{Mg}$ em relação ao Ni. Logo, a busca de uma tecnologia eficiente para a remoção seletiva de $\mathrm{Ca}$ e Mg presentes em soluções concentradas de Ni persiste, uma vez que a presença destas espécies interfere na etapa de eletrodeposição de $\mathrm{Ni}$ [12].

Nesse contexto, este trabalho propõe maximizar a extração seletiva de $\mathrm{Ca}$ e $\mathrm{Mg}$ do licor concentrado de Ni via extração por solventes utilizando-se o extratante Cyanex 272 e uma mistura sinérgica entre D2EHPA e ácido naftênico. Entende-se que o licor já passou pela etapa de pré-purificação para remoção de Zn, Co, Cu e Mn, conforme apresentado por Guimarães et al [8].

\section{MATERIAIS E MÉTODOS}

\subsection{Preparo das soluções aquosa e orgânica}

A fase aquosa foi preparada solubilizando-se sulfatos de $\mathrm{Ca}, \mathrm{Mg}$ e $\mathrm{Ni}$ (grau analítico, pureza 98\%, marca Synth) em água destilada. O licor sulfúrico obtido ([Ca] = 0,5 g/L, 
$[\mathrm{Mg}]=3,04 \mathrm{~g} / \mathrm{L}$ e $[\mathrm{Ni}]=88 \mathrm{~g} / \mathrm{L})$, concentrado em Ni em níveis similares aos dos licores industriais, foi homogeneizado em balão volumétrico e filtrado para remover eventuais sólidos em suspensão. $\mathrm{O}$ ajuste de $\mathrm{pH}$ foi realizado gotejando soluções de ácido sulfúrico (2 M; grau analítico; pureza 95\%; marca Synth) e/ou hidróxido de sódio (10 M; grau analítico; pureza 99\%; marca Synth).

As soluções orgânicas foram obtidas dissolvendo-se os extratantes D2EHPA (pureza 95\%; fabricante Lanxess; peso molecular: $322,43 \mathrm{~g} / \mathrm{mol}$; densidade a $20^{\circ} \mathrm{C}$ : $0,96 \mathrm{~g} / \mathrm{cm}^{3}$ ), Cyanex 272 (pureza 85\%; fabricante Cytec; peso molecular: $290 \mathrm{~g} / \mathrm{mol}$; densidade $\left.\left(24^{\circ} \mathrm{C}\right): 0,92 \mathrm{~g} / \mathrm{cm}^{3}\right)$ e ácido naftênico (pureza 97\%; marca Sigma-Aldrich; densidade $\left.\left(20^{\circ} \mathrm{C}\right): 0,92 \mathrm{~g} / \mathrm{cm}^{3}\right)$ em Exxsol D80 (diluente orgânico, fabricante Exxson, querosene alifático, com teor de aromáticos $\leq 0,5 \% \mathrm{p} / \mathrm{p}$ ).

\subsection{Ensaios de extração por solventes}

Os ensaios de extração por solventes foram conduzidos colocando, em um reator de vidro de $1 \mathrm{~L}$, iguais volumes das fases aquosa e orgânica ( $200 \mathrm{~mL}$ cada) a $50^{\circ} \mathrm{C}$. $\mathrm{O}$ reator foi posicionado em um banho-maria digital (Kacil, modelo RM-02). Com o auxílio de um agitador mecânico (450 rpm, Ika, modelo RW 20N), as soluções foram agitadas por 10 minutos, tempo suficiente para atingir o equilíbrio. Em seguida, a mistura foi deixada em repouso por 3 minutos com o intuito de se obter a separação das fases. $\mathrm{O}$ pH do licor sulfúrico foi medido com o auxílio de um pHmetro (Quimis, modelo 0400 AS). Alíquotas das soluções aquosa e orgânica foram recolhidas separadamente nos valores desejados de $\mathrm{pH}$, de modo a manter-se a razão entre os volumes das fases aquosa e orgânica unitária (razão $A / O=1$ ). Os ensaios cinéticos de extração por solventes foram realizados seguindo o mesmo procedimento experimental ora descrito, porém gotejou-se, com o auxílio de uma bureta, um volume determinado de solução de hidróxido de sódio (quantidade determinada mediante ensaio preliminar) almejando-se atingir o pH final desejado. Durante a agitação das fases, foram recolhidas alíquotas da mistura das soluções utilizando-se seringas nos seguintes intervalos de tempo (minutos): 0,5, 1, 1,5, 2, 3, 5 e 10.

As alíquotas recolhidas do licor foram adequadamente diluídas e analisadas quantitativamente por espectroscopia de absorção atômica (GBC, modelo XplorAA dual). Por balanço de massa, calculou-se a concentração dos metais em fase orgânica.

\section{RESULTADOS E DISCUSSÃO}

\subsection{Comportamento de extração dos metais $\mathrm{Ca}, \mathrm{Mg} \mathrm{e} \mathrm{Ni}$}

A Figura 1 mostra o comportamento de extração de $\mathrm{Ca}, \mathrm{Mg}$ e $\mathrm{Ni}$ contidos no licor sulfúrico utilizando-se: (i) mistura sinérgica entre D2EHPA $(10 \% \mathrm{v} / \mathrm{v})$ e ácido naftênico $(20 \% \mathrm{v} / \mathrm{v})$ e (ii) extratante Cyanex $272(20 \% \mathrm{v} / \mathrm{v})$, dissolvidos em Exxsol D80. 

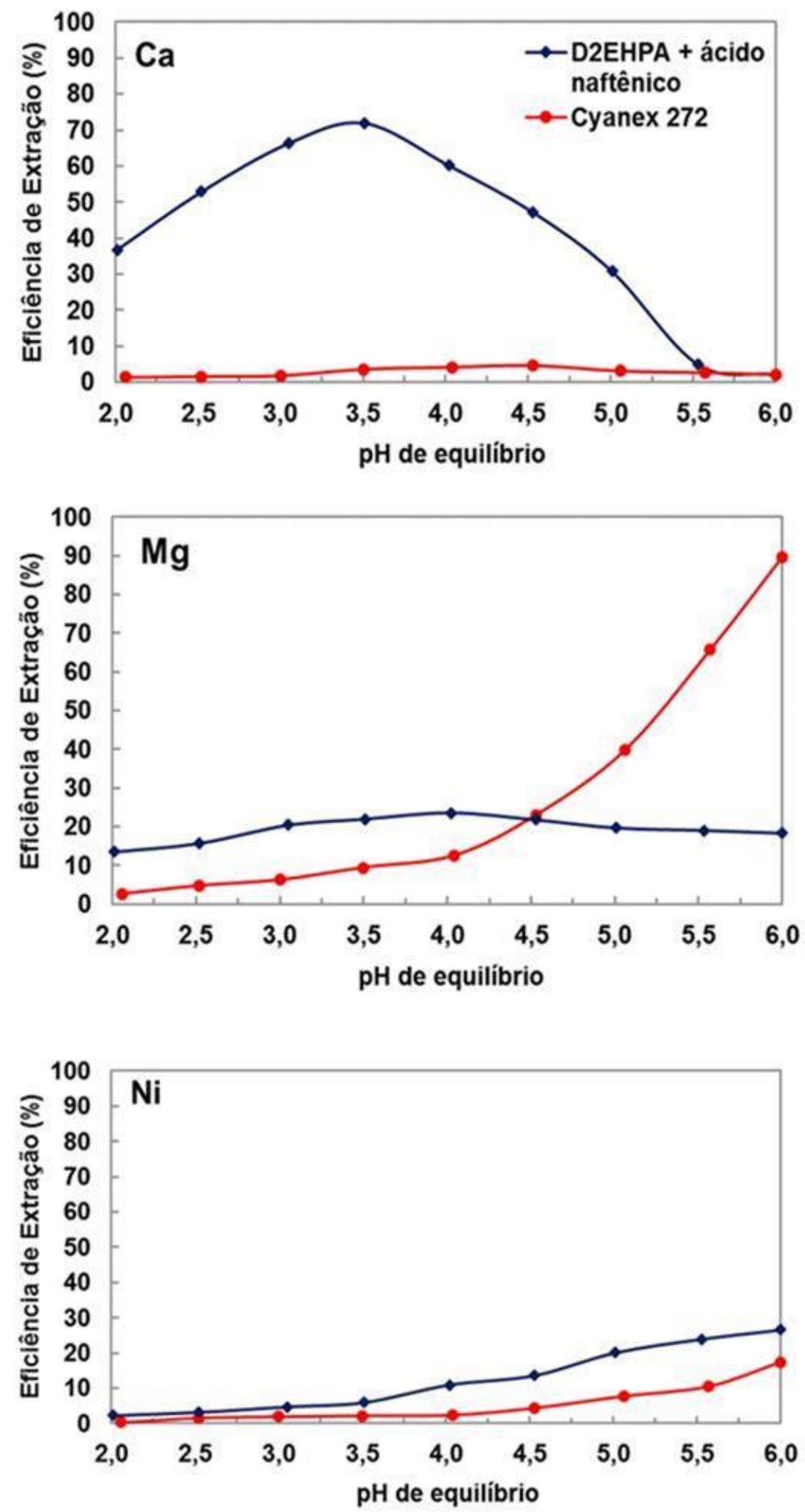

Figura 1. Curvas de extração percentual de $\mathrm{Ca}(0,5 \mathrm{~g} / \mathrm{L}), \mathrm{Mg}(3,04 \mathrm{~g} / \mathrm{L})$ e $\mathrm{Ni}(88 \mathrm{~g} / \mathrm{L})$ utilizando-se $10 \%$ v/v D2EHPA + 20\% v/v ácido naftênico (curva azul) e 20\% v/v Cyanex 272 (curva vermelha), dissolvidos em Exxsol D80, razão $\mathrm{A} / \mathrm{O}=1$ a $50^{\circ} \mathrm{C}$.

Observa-se que o sistema orgânico contendo D2EHPA + ácido naftênico possui maior afinidade na extração de Ca (Figura 1(a)). A seguinte ordem de extração foi obtida: $\mathrm{Ca}>\mathrm{Mg}>\mathrm{Ni}$ para $\mathrm{pH} \leq 5$. As extrações de $\mathrm{Ca}$ e de $\mathrm{Mg}$ aumentaram com a 
elevação do $\mathrm{pH}$ do licor e atingiram um máximo de $72 \%$ e $24 \%$ em $\mathrm{pH} 3,5$ e 4,0, respectivamente. Em seguida, as extrações de Mg decresceram gradativamente para $19 \%$ em $\mathrm{pH} 6$, enquanto as de $\mathrm{Ca}$ diminuíram progressivamente até atingir $1,5 \%$ em pH 5,5. Comportamento oposto é verificado em relação à preferência de extração dos metais alcalinos terrosos usando-se Cyanex 272. As extrações de $\mathrm{Mg}$ aumentaram com a elevação do $\mathrm{pH}$ do licor e atingiram $90 \% \mathrm{em} \mathrm{pH} 6$, e o Ca foi rejeitado pelo Cyanex 272 (extrações < 4,6\%) para $2 \leq \mathrm{pH} \leq 6$. Similaridades nas extrações de Ni foram obtidas para ambos os sistemas orgânicos (Figura 1(c)). Suas extrações aumentaram gradualmente com a diminuição de acidez do licor e atingiram em pH 6, 17\% e 26\%, utilizando-se os extratantes Cyanex 272 e D2EHPA + ácido naftênico, respectivamente.

Analisou-se por meio de ensaios cinéticos, mostrados na Figura 2, a existência de máximo nas curvas de extração de Ca e Mg (Figuras 1(a) e 1(b)).
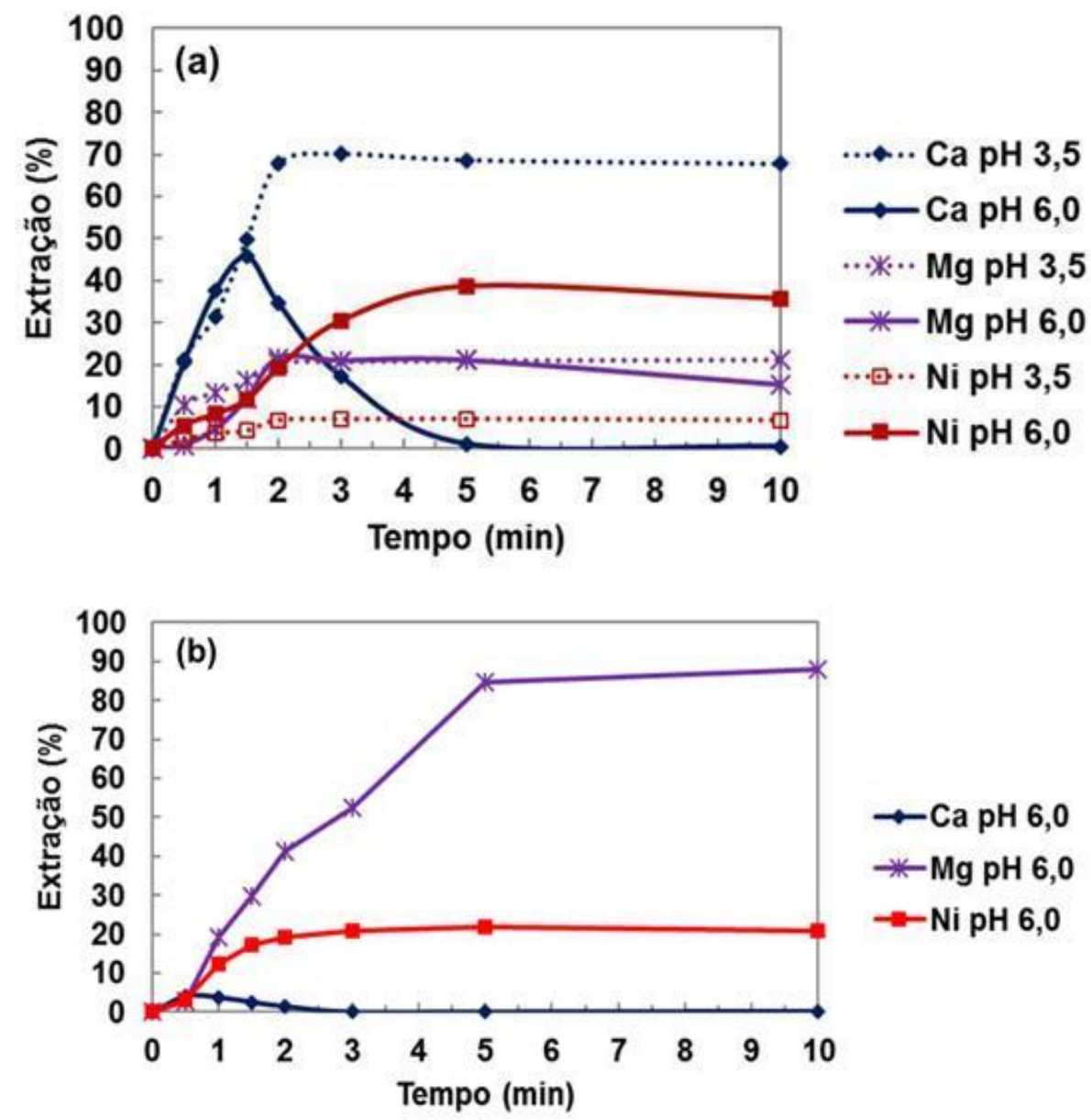

Figura 2. Extração cinética de $\mathrm{Ca}(0,5 \mathrm{~g} / \mathrm{L}), \mathrm{Mg}(3,04 \mathrm{~g} / \mathrm{L})$ e $\mathrm{Ni}(88 \mathrm{~g} / \mathrm{L})$ do licor sintético sulfúrico em pH 3,5 e 6,0 utilizando-se (a) 10\% v/v D2EHPA + 20\% v/v ácido naftênico, e (b) 20\% v/v Cyanex 272, dissolvidos em Exxsol D80, razão $\mathrm{A} / \mathrm{O}=1$ a $50^{\circ} \mathrm{C}$.

De maneira geral, observa-se que a cinética de extração dos metais pelo sistema D2EHPA + ácido naftênico é rápida, o que favorece sua aplicação em escala industrial. Em pH 3,5, o equilíbrio é alcançado em apenas 2 minutos, com extrações de aproximadamente $68 \%$ de $\mathrm{Ca}, 20 \%$ de $\mathrm{Mg}$ e $7 \%$ de $\mathrm{Ni}$. Em pH 6, há um aumento da competição metálica entre $\mathrm{Ca}, \mathrm{Mg}$ e $\mathrm{Ni}$ pelos extratantes D2EHPA e ácido naftênico, em função das maiores extrações de $\mathrm{Ni}$ (Figuras 1(c) e 2(a)). Como resultado das elevadas razões de concentração do $\mathrm{Ni}$ em relação ao $\mathrm{Ca}([\mathrm{Ni}] /[\mathrm{Ca}]=$ 
176) e $\mathrm{Mg}([\mathrm{Ni}] /[\mathrm{Mg}]=29)$, o Ni disponível em fase aquosa substitui o $\mathrm{Ca}$ e o $\mathrm{Mg}$ extraídos pelo sistema D2EHPA + ácido naftênico. Com isso, quase todo o $\mathrm{Ca}$ (cerca de $45 \%$ ) e parte do $\mathrm{Mg}(6 \%)$ retornam para a fase aquosa (licor) com 0 aumento do tempo (1,5 $\leq$ tempo $\leq 10$ minutos). Consequentemente, a extração percentual desses metais diminui, o que acarreta em pontos de máximo nas curvas de extração observadas para o Mg e, principalmente, para o Ca (Figuras 1(b) e 1(a), respectivamente). Esse fenômeno é conhecido por efeito crowding out e já foi observado na literatura especializada nas extrações de $\mathrm{Mn}$ e Co [4,10]. Portanto, infere-se que as separações $\mathrm{Ca} / \mathrm{Ni}$ e $\mathrm{Mg} / \mathrm{Ni}$ são favorecidas para $\mathrm{pH} \leq 3,5$ e $\mathrm{pH} \leq 4$, respectivamente, uma vez que o efeito crowding out não foi observado (Figuras 1(a), 1 (b) e 2(a)). Este nível de $\mathrm{pH}$ também é interessante, pois evita ajuste de $\mathrm{pH}$ no licor pré-tratado com Cyanex 272 para extração de Co, Mn, Cu e Zn. Em valores de pH > 3,5 e pH > 4, como resultado desse efeito, a separação do $\mathrm{Ca}$ e $\mathrm{Mg}$ em relação ao $\mathrm{Ni}$ é dificultada. Logo, deve-se evitar a purificação do licor nessas faixas de $\mathrm{pH}$. Verifica-se na Figura 2(b) que a extração de Mg pelo Cyanex 272, em pH 6, é rápida (equilíbrio foi alcançado em $5 \mathrm{~min}$ com cerca de $85 \%$ de extração) e não é prejudicada, uma vez que o efeito crowding out não é observado. Já a extração de Ca é afetada por esse efeito a partir de 30 segundos, indicando que a separação $\mathrm{Ca} / \mathrm{Ni}$ pelo Cyanex 272 é extremamente difícil.

\subsection{Fatores de separação do Ca e $\mathrm{Mg}$ em relação ao $\mathrm{Ni}$}

No contexto deste trabalho, os valores dos fatores de separação $\left(\beta_{\mathrm{M} / \mathrm{Ni}}\right)$ indicam o quão eficiente um extratante (ou mistura dos extratantes) pode separar seletivamente $\mathrm{M}$ (Ca ou $\mathrm{Mg}$ ) em relação ao $\mathrm{Ni}$. Esse fator pode ser calculado por meio da equação (1):

$$
\beta_{M} /_{N i}=\frac{D_{M}}{D_{N i}}=\frac{[M]_{\text {org }} /[M]_{a q}}{[N i]_{\text {org }} /[N i]_{a q}}(1)
$$

na qual $D_{M}\left(\right.$ ou $D_{N i}$ ) representa o coeficiente de distribuição. O valor de $D$ é calculado por meio da razão entre a concentração do metal $M$ nas fases orgânica e aquosa em cada $\mathrm{pH}$ de equilíbrio desejado [13]. Os valores dos fatores de separação $\beta \mathrm{Ca} / \mathrm{Ni}$ e $\beta_{\mathrm{Mg} / \mathrm{Ni}}$ são apresentados na Tabela 1.

Tabela 1. Fatores de separação $\beta_{\mathrm{Ca} / \mathrm{Ni}}$ e $\beta_{\mathrm{Mg} / \mathrm{Ni}}$ utilizando-se D2EHPA + ácido naftênico e Cyanex 272 , razão $\mathrm{A} / \mathrm{O}=1$ a $50^{\circ} \mathrm{C}$

\begin{tabular}{ccccc}
\hline \multirow{2}{*}{$\begin{array}{c}\text { pH de } \\
\text { equilíbrio }\end{array}$} & \multicolumn{2}{c}{$\begin{array}{c}\text { D2EHPA + ácido } \\
\text { naftênico }\end{array}$} & \multicolumn{2}{c}{ Cyanex 272 } \\
\cline { 2 - 5 } & $\boldsymbol{\beta}_{\mathrm{Ca} / \mathrm{Ni}}$ & $\boldsymbol{\beta}_{\mathrm{Mg} / \mathrm{Ni}}$ & $\boldsymbol{\beta}_{\mathrm{Ca} / \mathrm{Ni}}$ & $\boldsymbol{\beta}_{\mathrm{Mg} / \mathrm{Ni}}$ \\
\hline 2,5 & 25 & 7 & 1 & 3 \\
\hline 3,0 & 34 & 6 & 1 & 4 \\
\hline 3,5 & 40 & 5 & 2 & 5 \\
\hline 4,0 & 41 & 4 & 2 & 6 \\
\hline 4,5 & 12 & 3 & 1 & 7 \\
\hline 5,0 & 6 & 2 & 0,4 & 8 \\
\hline 5,5 & 2 & 1 & 0,2 & 16 \\
\hline 6,0 & 0,2 & 0,7 & 0,1 & 41 \\
\hline
\end{tabular}

De maneira geral, o desempenho do sistema D2EHPA + ácido naftênico favorece a

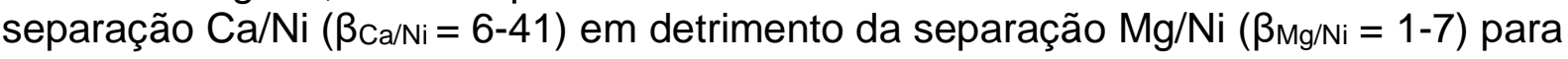


$2,5 \leq \mathrm{pH} \leq 6,0$. Como consequência do efeito crowding out, a seletividade desses extratantes, principalmente em relação ao $\mathrm{Ca}$, é comprometida para $\mathrm{pH}>4$, resultando em decréscimo nos valores de $\beta_{\mathrm{Ca} / \mathrm{Ni}}$ e $\beta_{\mathrm{Mg} / \mathrm{Ni}}$. Já a eficiência da seletividade do Cyanex 272 em relação ao Mg é notavelmente superior à do Ca na

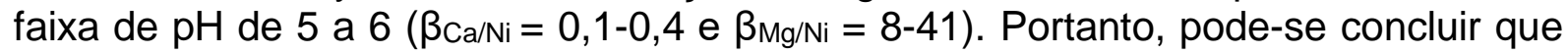
as separações $\mathrm{Ca} / \mathrm{Ni}$ e $\mathrm{Mg} / \mathrm{Ni}$ podem ser realizadas utilizando-se 2 circuitos de extração. No primeiro, Ca pode ser removido do licor pelo sistema D2EHPA + ácido naftênico. O pH ótimo é 3,5, uma vez que são extraídos cerca de $72 \%$ de Ca com apenas $6 \%$ de $\mathrm{Ni}$ apresentando $\beta_{\mathrm{Ca} / \mathrm{Ni}}=40$. Podem ser estimados 2 estágios de contatação (número de vezes que o licor deve ser colocado em contato com uma nova solução orgânica, isenta de metais, para promover a máxima extração dos metais de interesse) para a completa extração de $\mathrm{Ca}$ do licor. Em seguida, no segundo circuito, o licor deve ser direcionado a uma etapa de extração de Mg pelo Cyanex 272. Recomenda-se que essa extração seja conduzida em pH 5,5, no qual são removidos $66 \%$ de $\mathrm{Mg}\left(\beta_{\mathrm{Mg} / \mathrm{Ni}}=16\right)$ com $11 \%$ de $\mathrm{Ni}$, estimando-se de 2 estágios de contatação. Em ambos os circuitos, o Ni co-extraído com o $\mathrm{Ca}$ e $\mathrm{Mg}$ pode ser recuperado da fase orgânica por meio da técnica de reextração seletiva [14]. Logo, o licor obtido contendo Ni purificado em relação aos metais alcalinos terrosos pode ser direcionado a etapa de eletrorrecuperação obtendo-se catodos de $\mathrm{Ni}$.

\section{CONCLUSÕES}

- Ca é extraído preferencialmente pelo sistema orgânico contendo D2EHPA + ácido naftênico em $\mathrm{pH} \leq 5$ e Mg pelo Cyanex 272 em $5 \leq \mathrm{pH} \leq 6$;

- A cinética de extração de Ca e Mg é rápida alcançando o equilíbrio em tempo inferior a 6 minutos;

- O efeito crowding out observado para o sistema D2EHPA + ácido naftênico, compromete as separações $\mathrm{Ca} / \mathrm{Ni}$ para $\mathrm{pH}>3,5$ e $\mathrm{Mg} / \mathrm{Ni}$ para $\mathrm{pH}>4$;

- A purificação do licor concentrado de Ni requer o uso de 2 circuitos de extração. O sistema D2EHPA + ácido naftênico deve ser utilizado para promover a extração de $\mathrm{Ca}$. A melhor condição para essa finalidade ocorre em $\mathrm{pH} 3,5\left(\beta_{\mathrm{Ca} / \mathrm{Ni}}=40\right)$, no qual são extraídos $72 \%$ de $\mathrm{Ca}$ com $22 \%$ de $\mathrm{Mg}$ e $6 \%$ de Ni. Estima-se 2 estágios de contatação para alcançar a máxima extração de $\mathrm{Ca}$;

- O licor isento de Ca pode ser submetido a uma extração de Mg pelo Cyanex 272. O pH ótimo é 5,5 , em que são extraídos $66 \%$ de $\mathrm{Mg}\left(\beta_{\mathrm{Mg} / \mathrm{Ni}}=16\right) \mathrm{com}$ $11 \%$ de $\mathrm{Ni}$. São estimados 2 estágios de contatação para a completa remoção de $\mathrm{Mg}$ do licor. A solução aquosa purificada de $\mathrm{Ni}$ pode ser direcionada à etapa de eletrorrecuperação obtendo-se Ni metálico.

\section{Agradecimentos}

Os autores agradecem pelo suporte financeiro do CNPq (CT-Mineral, Processo 550243/2011-9, bolsa Produtividade em Pesquisa n $304050 / 2016-4$ e bolsa de doutorado), FAPEMIG, CAPES-PROEX e INCT-Acqua (Instituto Nacional de Ciência e Tecnologia em Recursos Minerais, Água e Biodiversidade www.acqua-inct.org), e também à Cytec Canadá pelo fornecimento de amostras de Cyanex 272.

\section{REFERÊNCIAS}


1 Williams $\mathrm{C}$, William $\mathrm{H}$, Vaughan J. Selective leaching of nickel from mixed nickel cobalt hydroxide precipitate. Hydrometallurgy. 2013; 138: 84-92.

2 Chong S, Hawker W, Vaughan J. Selective reductive leaching of oxidized cobalt containing residue. Minerals Engineering. 2013; 54: 82-87.

3 Sole K, Cole P. Purification of nickel by solvent extraction. Solvent Extraction and Ion Exchange. 2003; 15: 143-195.

4 Cheng CY, Urbani MD, Davies MG, Pranolo Y, Zhu, Z. Recovery of nickel and cobalt from leach solutions of nickel laterites using a synergistic system consisting of Versatic 10 and Acorga CLX 50. Minerals Engineering. 2015; 77: 17-24.

5 Innocenzi V, Vegliò F. Separation of manganese, zinc and nickel from leaching solution of nickel-metal hydride spent batteries by solvent extraction. Hydrometallurgy. 2012; 129-130: 50-58.

6 Sousa Junior CS, Ximenes DS, Nascimento M, Nascimento KRPR, Cunha OGC. Modelagem termodinâmica por extração por solvente de metais divalentes em meio sulfato usando D2EHPA. Química Nova. 2012; 35(2): 301307.

7 Ndlovu B, Mahlangu T. Calcium and magnesium rejection from sulphate solutions in lateritic nickel solvent extraction using Versatic 10 acid-LIX ${ }^{\circledR} 84$ IC system. Template Journal. 2008; 108: 223-227.

8 Guimarães AS, Silva PS, Mansur, MB. Purification of nickel from multicomponent aqueous sulfuric solutions by synergistic solvent extraction using Cyanex 272 and Versatic 10. Hydrometallurgy. 2014; 150: 173-177.

9 Donegan S. Direct solvent extraction of nickel at Bulong operations. Minerals Engineering. 2006; 19: 1234-1245.

10 Cheng, CY. Purification of synthetic laterite leach solutions by solvent extraction using D2EHPA. Hydrometallurgy. 2000; 56: 369-386.

11 Guimarães AS, Mansur MB. Extração por solventes sinérgica de níquel, cálcio e magnésio com os extratantes Cyanex 272 e Cyanex 923. In: Encontro Nacional de Tratamento de Minérios e Metalurgia Extrativa; 2015; Poços de Caldas, Brasil. Minas Gerais: ENTMME; 2015. p. 910-918.

12 Santos LH, Carvalho PLG, Rodrigues GD, Mansur MB. Selective removal of calcium from sulfate solutions containing magnesium and nickel using aqueous two phase systems (ATPS). Hydrometallurgy. 2015; 156: 259-263.

13 Babakhani A, Rashchi F, Zakeri A, Vahidi E. Selective separation of nickel and cadimium from sulfate solutions of spent nickel-cadmium batteries using mixtures of D2EHPA and Cyanex 302. Journal of Power Sources. 2014; 247: 127-133.

14 Wang LY, Lee MS. Development of a separation process for the selective extraction of hafnium(IV) over zirconium(IV) from sulfuric acid solutions by using D2EHPA. Hydrometallurgy. 2016; 160: 12-17. 